\title{
Research about rolling element bearing fault diagnosis based on mathematical morphology and sample entropy

\author{
Lingli Cui ${ }^{1, a^{*}}$, Xiangyang Gong ${ }^{1, b}$ and Yu Zhang ${ }^{1, c}$
}

${ }^{1}$ Beijing Engineering Research Center of Precision Measurement Technology and Instruments, Beijing University of Technology, Beijing, China

\author{
aacuilingli@163.com, bogngxiangyang6@163.com, cyuzh_2014@163.com
}

Keywords: mathematical morphology; pattern spectrum; sample entropy; BP neural network Abstract. In view of the non-linear and non-stationary of the rolling element bearing fault signal, the method of mathematical morphology analysis is introduced into the rolling element bearing fault diagnosis. Multi-scale morphological transform is applied to the analysis of the bearing signals. To describe the complexity of pattern spectrum curves by using sample entropy, and its value as the input vector of the neural network is used to realize the fault pattern classification by using the back-propagation (BP) neural network. Experimental results show that this method is effective.

\section{Introduction}

Rolling element bearing is one of the most widely components used in the transmission machinery. So the bearing fault diagnosis has a great significance. The signals generated by the bearing fault are non-linear and non-stationary signals, so the traditional fault diagnosis methods have the limitation to process the signals. As a nonlinear analysis method, mathematical morphology is very suitable to analysis the bearing signals. Hao at al [1] proposed a novel method based on pattern spectrum entropy and support vector machine (SVM). The method showed that this method was effective to realize the bearing fault diagnosis. According to the work by Chen, a new double-dot structuring element (SE) is constructed to analysis the bearing fault signals [2]. The result of the above studies have verified the validity of the mathematical morphology in mechanical fault diagnosis, but the effect of mathematical morphology analysis is often influenced by the morphological operators and the structural elements which need to be the further researched. The pattern spectrum of the signals can be obtained by using the mathematical morphology analysis which can reflect the morphological changes of the signals. Therefore, a novel method of rolling element bearing fault diagnosis based on the mathematical morphology and sample entropy is proposed.

\section{Multi-scale morphology and pattern spectrum}

The key of mathematical morphology analysis is the selection of structural element and morphological operator. Compared to the length of the structure element, the effect of the shape of the structure element is relatively small to analyze the signals. Therefore, a simple linear structure element is adopted to reduce the computation in this paper. In the multi-scale morphological transformation, the size of the structure element would change with the scale.

The concept of the pattern spectrum was proposed by P.Maragos[3]. Assumption the $f(x), x \in R^{m}, m=1,2, \ldots, M-1$ is a non negative function, and $\mathrm{M}$ is a natural number. $g(x)$ is a convex structure function. Then the pattern spectrum of $f(x)$ can be defined as follows:

$$
\begin{array}{ll}
P S(f, g, \lambda)=\frac{-d A(f \Delta \lambda g)}{d \lambda} & \lambda \geq 0 \\
P S(f, g, \lambda)=\frac{d A(f *(-\lambda g)}{d A} & \lambda<0
\end{array}
$$

Where $A(f)=\int f(x) \mathrm{dx}$ denotes the limited area of the umbra in the definition domain.

When $\lambda \geq 0$, it is the opening operation pattern spectrum. On the contrary, it is the opening operation pattern spectrum. The pattern spectrum curve means that the morphological character varies as the continuous changing of the scale. 


\section{Analysis of experimental data}

In order to verify the feasibility of the algorithm in this paper, the measured signal data of the bearing in the Key Laboratory of Advanced Manufacturing Technology of Beijing University of Technology is analyzed. The type of fault bearing is SKF 6307, and the rotation speed of the shaft is 1496rpm with the sampling frequency of $12.8 \mathrm{kHz}$. The bearing test rig is shown in Fig. 1.

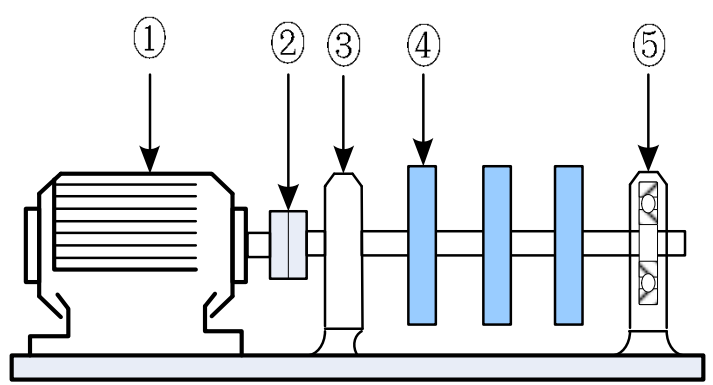

Fig. 1 Bearing test rig

According to the definition of the mathematical morphology and pattern spectrum, the vibration signals with different fault types are processed. The time-domain and pattern spectrum of the fault signals are shown in Fig. 2.

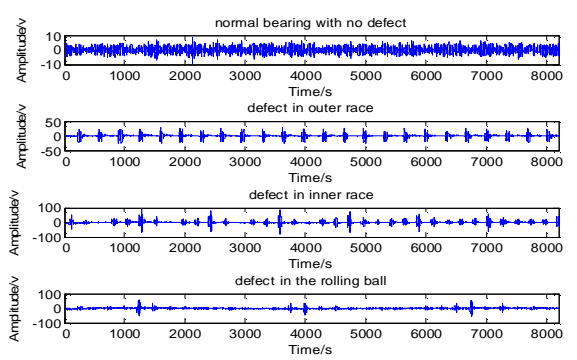

(a)Time domain waveform of different signals

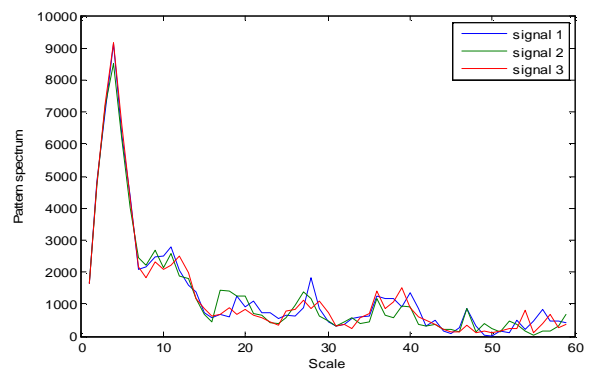

(c) Pattern spectrum of outer race fault

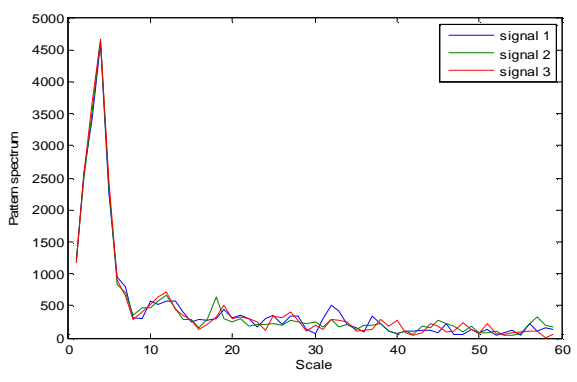

(e) Pattern spectrum of rolling body fault

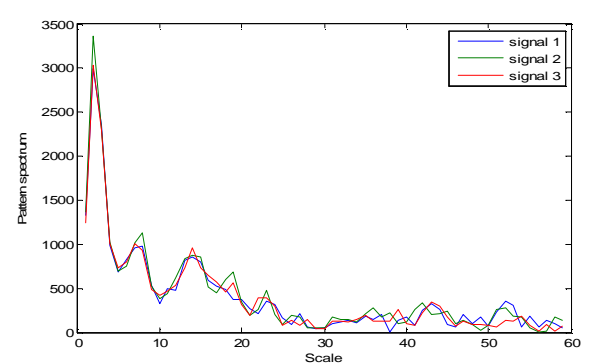

(b) Pattern spectrum of normal bearing

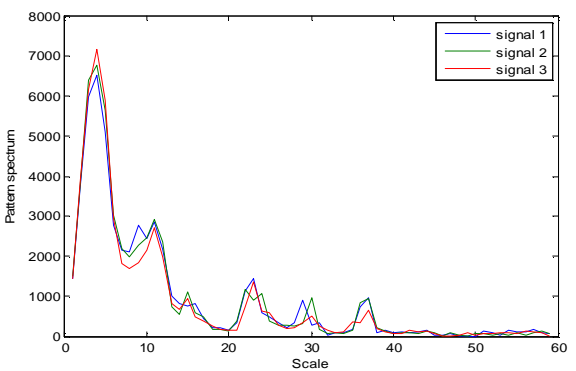

(d) Pattern spectrum of inner race fault

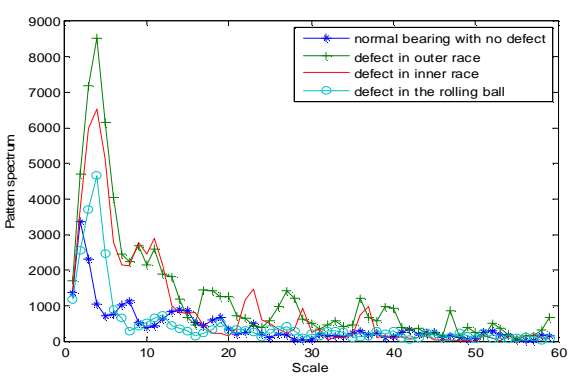

(f) Pattern spectrum of different fault signals

Fig. 2 Time-domain and pattern spectrum of the fault signals 
From the figure (b), (c), (d), (e), it can be seen that the pattern spectrum curves of the bearing signals with common fault type are similar in shape and trend. The scale of the structural element corresponded the larger amplitude of the curve is similar, and it only has a slight difference in the amplitude. From the figure (f), it can be seen that the pattern spectrum curves of the three types fault signals are different with the normal bearing signals. The curve of the normal bearing reaches the maximum value when the scale is 2 , and then the curve tends to be flat. But the curves of the fault bearing reach the maximum value when the scale is 4 . The shape spectrum curves of different fault types are different, and the maximum amplitudes are also different. When the amplitude of the shape spectrum curve is large, the signal characteristic information of the signals matched the structure element in the scale is more.

\section{Quantitative analysis of pattern spectrum curve}

The method of mathematical morphology analysis can reach the purpose of distinguishing the distribution characteristic of the different fault signals. But it is not obvious to recognize the different fault types. In order to describe the pattern spectrum curves of different bearing faults, the definition of sample entropy is introduced in this paper.

According to the sample entropy algorithm[4], the values of the pattern spectrum curves with different fault are calculated. The sample entropy values of pattern spectrum curves are shown in Table1.

Table1 The sample entropy of pattern spectrum curves

\begin{tabular}{ccccc}
\hline Serial number & Normal & $\begin{array}{c}\text { Outer race } \\
\text { fault }\end{array}$ & $\begin{array}{c}\text { Inner race } \\
\text { fault }\end{array}$ & Rolling body fault \\
\hline 1 & 0.432 & 1.048 & 1.458 & 0.611 \\
2 & 0.459 & 1.131 & 1.620 & 0.637 \\
3 & 0.396 & 1.115 & 1.575 & 0.605 \\
4 & 0.529 & 1.007 & 1.601 & 0.644 \\
5 & 0.541 & 1.025 & 1.550 & 0.647 \\
6 & 0.413 & 1.097 & 1.548 & 0.562 \\
7 & 0.416 & 1.089 & 1.572 & 0.572 \\
8 & 0.308 & 1.036 & 1.661 & 0.792 \\
9 & 0.501 & 0.991 & 1.374 & 0.540 \\
10 & 0.517 & 1.097 & 1.593 & 0.596 \\
\hline
\end{tabular}

\section{BP neural network recognition of bearing fault type}

In recent years, the application of BP neural network in the fault diagnosis of rolling element bearing is widely studied[5]. In this paper, the inputs of BP neural network are the sample entropy values of the vibration signals with different fault types. Twenty five sets of experimental data for each fault are selected for training, and the sample number is 100 . The corresponding relationship between the network outputs and fault types is shown in Table 2.

In order to research the performance of the network, the 25 groups of experiment signals with each fault type are used to verify. Table 3 shows the verification results of network. From the Table 3, it can be seen that the ideal results are obtained by combining the multi-scale morphological analysis with BP neural network in the pattern recognition of the bearing fault types.

Table2 Mapping results between net outputs and fault types

\begin{tabular}{|c|c|c|}
\hline Serial number & Ideal output & Fault type \\
\hline 1 & 1000 & Normal \\
\hline 2 & 0100 & Outer race fault \\
\hline 3 & 0010 & Inner race fault \\
\hline 4 & 00001 & Rolling body fault \\
\hline
\end{tabular}


Table3 Verification results of network

\begin{tabular}{cccc}
\hline Type of output & Number of samples & $\begin{array}{l}\text { Number of } \\
\text { recognition }\end{array}$ & Accuracy ratio \\
\hline 1 & 25 & 24 & $96 \%$ \\
2 & 25 & 23 & $92 \%$ \\
3 & 25 & 23 & $92 \%$ \\
4 & 25 & 24 & $96 \%$ \\
\hline
\end{tabular}

\section{Conclusions}

(1) A new back-propagation neural network method based on the Multi-scale morphology and sample entropy is proposed to realize the fault diagnosis of rolling element bearing. Morphological characteristics of bearing vibration signals in different scales can be reflected by the pattern spectrum. Quantitative identification of morphological spectrum curves can be realized by using the sample entropy method. And its value as the input vector of the neural network is used to realize the fault pattern classification by using the BP neural network.

(2) According to the proposed method in this paper, single-point pitting damage signals with different faults are analyzed. The pattern spectrum curves of bearing vibration signals with same fault are similar. On the contrary, the curves are different. A high recognition rate is got by using the BP neural network method. The result verifies the feasibility of the method.

\section{Acknowledgements}

This work is supported by the National Natural Science Foundation of China (Grant No.51175007 and 51575007 ) and Research base construction project of Beijing Municipal Commission of Education(PXM2015_014204_500002). Finally, the authors are grateful to the editors and anonymous reviewers for their helpful comments and constructive suggestions.

\section{References}

[1] Rujiang Hao, Zhike Peng, Zhipeng Feng. Application of support vector machine based on pattern spectrum entropy in fault diagnostics of rolling element bearings. Measurement

Science\&Technology, 2011, 22(4): 045708.

[2] Qiong Chen, Zhaowen Chen, et al. A new structuring element for multi-scale morphology analysis and its application in rolling element bearing fault diagnosis. Journal of Vibration and Control, 2015, 21(4):765-789.

[3] Maragos P. Pattern spectrum and multi-scale shape representation. IEEE Transactions on Pattern Analysis and Machine Intelligence, 1989. 11 (7): 701-716.

[4] Richman J S, Moorman R J. Physiological time-series analysis using approximate entropy and sample entropy. Am J Physiol Heart Circ Physiol, 2000, 278(6): 2039-2049.

[5] Jayaswal P, Verma S N, Wadhwani A K. Application of ANN, fuzzy logic and wavelet transform in machine fault diagnosis using vibration signal analysis. Journal of Quality in Maintenance Engineering, 2010, 16(2): 190-213. 